\title{
Borderline? Oder doch eher hochsensibel?
}

\author{
Sandra Konrad
}

Online publiziert: 10. Dezember 2020

(C) Der/die Autor(en) 2020

Zusammenfassung Menschen mit Hochsensibilität (SPS; Aron und Aron 1997) und einer BorderlinePersönlichkeitsstörung (BPS) weisen, basierend auf ihrem Temperament, Gemeinsamkeiten in Bezug auf ihre hohe Empfindsamkeit und Verletzlichkeit auf. Beide Personenkreise zeigen beispielsweise Beziehungsängste und Sorge vor interpersoneller Zurückweisung. Erste Hinweise auf einen Zusammenhang zwischen einer BPS und SPS liefert eine Studie von Meyer et al. (2005). Im Zeitraum 2015-2016 wurden im deutschsprachigen Raum $N=1105$ Erwachsene (MW-Alter 39,75 Jahre; $S D=11,50,954$ Frauen) online $\mathrm{zu}$ ihrer Feinfühligkeit und ihren BPS Symptomen befragt. SPS wurde mit der validierten deutschen Fassung der High Sensitiv Person Scale (HSPS-G) erhoben. Die BPS Symptome wurden mithilfe der Subskala Borderline (BL) des Persönlichkeits-Stil- und Störungs-Inventar (PSSI) erfasst. Die Korrelationen zwischen PSSI-BL und SPS zeigten schwache bis mittlere Zusammenhänge, jedoch waren die Zusammenhänge nach Auspartialisierung der jeweils anderen beiden Subskalen der HSPS-G mit BL (spontanerBorderline-Typus) geringer und finden sich eher im Zusammenhang mit dem Faktor EOE (Leichte Erregbarkeit) wieder. Im Gruppenvergleich berichten hochsensible Menschen höhere Werte auf der PSSIBL-Subskala als Nicht-Hochsensible. Da BPS und SPS Überschneidungspunkte aufweisen, sollte SPS bei BPS gegebenenfalls mit in Erwägung gezogen werden. Behandlungsimplikationen nach kognitiv-behavioralen Ansätzen werden vorgeschlagen.

S. Konrad $(\bowtie)$

Hochschule für Ökonomie und Management, Hamburg, Deutschland

konrads@hsu-hh.de
Schlüsselwörter BPS · Hochsensibilität . Sensory-processing sensitivity · Komorbidität · Differentialdiagnostik

\section{Borderline? Or rather highly sensitive?}

Summary Individuals with high sensory-processing sensitivity (SPS) and borderline personality disorder (BPS) share commonalities in terms of high sensitivity and vulnerability. Both groups show, for example, fear of relationships and concern about interpersonal rejection. A study by Meyer et al. (2005) provides initial indication of a connection between BPS and SPS. In the period 2015-2016, N=1105 adults ( $M$ age 39,75 years; $S D=11,50,954$ women) in Germanspeaking countries were surveyed online about their sensitivity and BPS symptoms. SPS was assessed using the German version of the High Sensitive Person Scale (HSPS-G). BPS symptoms were measured using the Borderline (BL) subscale of the Personality Style and Disorder Inventory (PSSI). Correlations between PSSI-BL and SPS showed weak to medium correlations. However, when partialling out the other two subscales of the HSPS-G the correlations were smaller and mainly remained for the factor Ease of Excitation (EOE). Group comparisons showed that individuals with SPS reported more BPS symptoms compared to a group without SPS. Regressions showed that EOE of the HSPS-G and age were the best predictors of BPS. As BPS and SPS have overlapping points, SPS should be considered for BPS where appropriate. Treatment implications according to cognitive-behavioural approaches are suggested.

Keywords BPS · Sensory-processing sensitivity • Comorbidity $\cdot$ Differential diagnosis 


\section{Borderline? Oder doch eher Hochsensibel?}

Frau S. hat zahlreiche Probleme. Sie errötet sehr leicht, wenn ihr etwas unangenehm ist oder ihr jemand zu nahe tritt. Soziale Kontakte sind für sie sehr anstrengend und sie fühlt sich häufig unverstanden und nicht angenommen, was dazu führt, dass sie sich zurück zieht. Sie wechselt häufig den Job, weil der Kontakt mit den Kollegen und Kolleginnen für Frau S. sehr nervenaufreibend und äußerst belastend ist. Sie erlebt sich ständig in einer Verteidigungshaltung und versucht mögliche und tatsächliche Angriffe abzuwehren. Dann flüchtet sie sich in Tagträume und kann ihre Aufgaben nicht mehr gut erledigen. Ihre Beziehungen enden nach kurzer Zeit, unter anderem weil sie Schwierigkeiten hat, ihrem Partner mehr Freiraum einzuräumen. Sie fühlt sich oft traurig und ängstlich und hat Stimmungsschwankungen. Ein schüchterner und emotional labiler Mensch könnte ein typisches Beispiel für einen Erwachsenen mit einer Borderline-Persönlichkeitsstörung (BPS) sein. Diese Beschreibung könnte jedoch genauso gut auf eine Person mit hoher sensorischer Verarbeitungssensitivität hindeuten.

Menschen unterscheiden sich darin, wie sie Umweltreize wahrnehmen und verarbeiten (Environmental Sensitivity). Unter Environmental Sensitivity lassen sich verschiedene Theorien subsumieren, welche sich alle mit der Sensitivität für Umweltreize beschäftigen. Dazu gehören beispielsweise Begrifflichkeiten wie Differential Susceptibility (Belsky 1997; Belsky und Pluess 2009), Biological Sensitivity to Context (Ellis et al. 2011) und die Sensory Processing Sensitivity (Aron und Aron 1997). All diesen Theorien ist gemein, dass sie sich mit der Reaktivität oder auch Ansprechbarkeit auf Reize beschäftigen und darin übereinstimmen, dass sich Menschen hinsichtlich ihrer Wahrnehmung und Verarbeitung von Stimuli unterscheiden, unabhängig davon, ob es sich um positive oder aversive Stimuli handelt (Greven et al. 2019).

\section{Hohe sensorische Verarbeitungssensitivität}

Personen mit hoher sensorischer Verarbeitungssensitivität (auch Hochsensible Personen (HSP) genannt) nehmen Reize intensiver wahr und verarbeiten diese auch anders, was in der Folge leicht zu einer Überstimulation und Überreizung führen kann. Dabei ist es unerheblich, ob die Reize interner oder externer Natur sind. Das Konzept basiert auf vier Indikatoren, die für eine Hochsensibilität oder „sensory-processingsensitivity“ (SPS) sprechen (Aron und Aron 1997) Dazu gehören: 1. Verhaltenshemmung/Rückzug, 2. tiefere Verarbeitung von sensorischen Informationen, 3. Sensitivität auf Stimuli und 4. emotionale/physiologische Reaktivität (Aron et al. 2012).

Zur Messung des Merkmals SPS schlagen Aron und Aron (1997) die High Sensitive Person-Skala (HSPS) vor. Allerdings war deren eindimensionale faktorielle Struktur in der Vergangenheit umstritten (ein Überblick in Konrad und Herzberg 2017). Jedoch konnte eine drei-faktorielle Struktur, bestehend aus den Faktoren „Leichte Erregbarkeit“ (Ease of Excitation, EOE), „Ästhetische Sensitivität“ (AES) und „Niedriger sensorischer Reizschwelle“ (LST) durch mehrere Autoren gezeigt werden (z. B. bei Evers et al. 2008; Konrad und Herzberg 2017; Liss et al. 2008; Smolewska et al. 2006). Während EOE für die emotionale Reaktivität auf physischen Input steht, misst AES die tiefere Verarbeitung im Sinne einer stärkeren Reflektion und einem höheren Bewusstsein. Der Faktor LST bildet die niedrige sensorische Reizschwelle ab (Konrad und Herzberg 2017). Damit finden sich drei der vier Indikatoren in den Faktoren wieder, Verhaltenshemmung/ Rückzug wird nicht separat, sondern durch den Faktor EOE abgebildet (Konrad und Herzberg 2017).

Nach Kagan et al. (1994) lassen sich ca. $20 \%$ aller Menschen als hochsensibel beschreiben, wobei neuere Studien auf einem prozentualen Anteil von 31\% sprechen (Lionetti et al. 2018). Trotz des hohen prozentualen Anteils von Betroffenen sind die empirische Belegung und das Wissen über das Konstrukt vergleichsweise gering.

\section{Borderline Persönlichkeitsstörung}

Persönlichkeitsstörung ist lediglich ein Oberbegriff, der viele unterschiedliche Arten von Störungen beinhaltet. Für die Einteilung werden hauptsächlich zwei unterschiedliche Systeme genutzt: die Internationale statistische Klassifikation der Krankheiten und verwandter Gesundheitsprobleme (ICD - aus dem Englischen International Statistical Classification of Diseases and Related Health Problems) oder das Diagnostic and Statistical Manual of Mental Disorders (DSM). Gemäß APA ist eine Persönlichkeitsstörung definiert als „ein überdauerndes Muster von innerem Erleben und Verhalten, das merklich von den Erwartungen der soziokulturellen Umgebung abweicht.“ Dieses Muster manifestiert sich in mindestens zwei der folgenden Bereiche: Kognition, Affektivität, Gestaltung zwischenmenschlicher Beziehungen und Impulskontrolle. Ferner geht man davon aus, dass dieses „Muster unflexibel und tiefgreifend in einem weiten Bereich persönlicher und sozialer Situationen“ ist und „in klinisch bedeutsamer Weise zu Leiden oder Beeinträchtigung in soziale, beruflichen oder anderen wichtigen Funktionsbereichen" führt. Dieses „Muster ist stabil und langdauernd, und sein Beginn ist zumindest bis in die Adoleszenz oder ins frühe Erwachsenenalter zurückzuverfolgen.“

Nach DSM V ist die Borderline Persönlichkeitsstörung (BPS) durch ein tiefgreifendes Muster von Instabilität in den Bereichen zwischenmenschlicher Beziehungen, im Selbstbild und in den Affekten sowie von deutlicher Impulsivität geprägt (American Psychiatric Association 2015). 
Das ICD-10 (ICD-10-GM; (Dilling et al. 2000)) unterscheidet zwei Formen der BPS, die Emotional instabile Persönlichkeitsstörung vom Impulsiven Typus (F60.30) und die Emotional instabile Persönlichkeitsstörung vom Borderline Typus (F60.31). Beiden Formen basieren auf einer emotionalen Instabilität, unterscheiden sich aber hinsichtlich ihrer Impulsivität.

\section{HSP und BPS}

Für die Beschreibungsdimensionen von Menschen mit SPS und BPS ergeben sich einige Gemeinsamkeiten: Beide zeigen eine Hypersensitivität für emotionale Reize, erhöhte Intensivität emotionalen Erlebens und eine verlangsamte Rückkehr zur emotionalen Baseline. Beide Konzepte basieren auf einer gewissen Reizoffenheit und einem daraus folgenden Risiko für eine Überstimulation, was durch eine höhere Vulnerabilität basierend auf dem Temperament begründet sein könnte (Meyer et al. 2005). Allerdings weisen nicht alle Hochsensiblen diese erhöhte Vulnerabilität auf, sondern nur die, die Verarbeitungsprobleme zeigen (Hinterberger et al. 2019).

Auch wird für beide Konzepte eine moderate bis hohe Heritabilität angenommen (Assary et al. 2020; Torgersen et al. 2000) und es besteht eine hohe Komorbidität an einer psychischen Störung (z. B. Angst, Depression) zu erkranken (z. B. Ahadi und Basharpoor 2010; Eaton et al. 2011; Konrad und Herzberg 2017).

Menschen mit BPS leiden unter niedrigem Selbstwertgefühl (Lammers et al. 2007), geringerem Bildungserfolg, weniger beruflichem Erfolg (Khayal et al. 2016) oder auch weniger erfolgreichen Beziehungen (z. B. Stepp et al. 2009; Russell et al. 2007). Für hochsensible Menschen trifft dies ebenso $\mathrm{zu}$, vor allem, wenn sie in einem wenig unterstützenden Umfeld aufgewachsen sind (Aron und Aron 1997). So fanden Aron und Aron (1997) in ihren Studien zwei verschiedene Gruppen hochsensibler Personen, zum einen eine kleinere Gruppe (etwa ein Drittel der TeilnehmerInnen), die angaben, eine unglückliche Kindheit gehabt $\mathrm{zu}$ haben und tendenziell höhere Werte für soziale Introversion und Emotionalität aufwiesen und zum anderen eine größere Gruppe (die anderen zwei Drittel der TeilnehmerInnen), welche sich nur hinsichtlich ihrer Grundsensibilität von der Population nicht hochsensibler Personen unterschieden. In einer weiteren Untersuchung (Aron et al. 2005) stellten sie fest, dass Schüchternheit im Erwachsenenalter durch eine Interaktion von Hochsensibilität und schlechten Kindheitserfahrungen häufiger auftritt, als wenn NON-HSP schlechte Kindheitserfahrungen berichteten. Ferner konnte auch festgestellt werden, dass auch negative Affekte im Erwachsenenalter durch diese Interaktion begünstigt werden können (Aron Aron et al. 2005).

Trotz der Gemeinsamkeiten im Verhalten von Menschen mit SPS und BPS gibt es auch zentrale Unterschiede. Bei Menschen mit BPS liegt eine „deut- liche Tendenz, Impulse ohne Berücksichtigung von Konsequenzen auszuagieren, verbunden mit unvorhersehbarer und launenhafter Stimmung " vor. Ferner „besteht eine Neigung zu emotionalen Ausbrüchen und eine Unfähigkeit, impulshaftes Verhalten zu kontrollieren“ und „eine Tendenz zu streitsüchtigem Verhalten und zu Konflikten mit anderen, insbesondere wenn impulsive Handlungen durchkreuzt oder behindert werden“ (ICD-10-GM; (Dilling et al. 2000)). Dieses Verhalten lässt sich bei hochsensiblen Menschen eher nicht wiederfinden. Hochsensible tendieren eher dazu, erst zu überlegen, bevor sie agieren (Aron und Aron 1997; Aron et al. 2012). Auch die Impulsivität, die kennzeichnend für den impulsiven Subtyp der BPS (F60.30) ist, findet sich bei SPS nicht (Aron und Aron 1997; Aron et al. 2012), was eher für den instabilen Subtyp der BPS (F60.31) bei hochsensiblen Menschen sprechen würde. Anders als Menschen mit BPS reflektieren Hochsensible und überlegen länger, bevor sie handeln (Patterson und Newman 1993; zit. nach Aron und Aron 1997). Die Gemeinsamkeiten auf der Verhaltensebene könnten somit auf gemeinsame Defizite in Neurotransmitterhaushalten oder genetischen Überschneidungen zurückzuführen sein.

\section{Zentrale Fragestellung}

Der Zusammenhang von SPS und einem Borderline Persönlichkeitsstil soll empirisch geprüft werden. Hierbei soll herausgearbeitet werden, inwieweit sich beide Phänomene, trotz phänotypischer Gemeinsamkeiten, unterscheiden und wie sie voneinander abgegrenzt werden können. Ein Gruppenvergleich soll Gemeinsamkeiten und Unterschiede zwischen Hochsensiblen (HSP) und Nicht-Hochsensiblen (NONHSP) in ihrer Borderline Symptomatik untersuchen. Die Regressionsanalyse ist ein nützliches Verfahren für Prognosen. Mit ihr soll geprüft werden, wie gut die Variable SPS geeignet ist, einen Borderline Persönlichkeitsstil vorherzusagen. Anhand der vorliegenden Ergebnisse soll eine erste Abgrenzung zwischen beiden Konstrukten vorgenommen werden.

Möglicherweise bestehende Alters- und Geschlechtseffekte sollen mit berücksichtigt werden.

\section{Methoden}

\section{Protokoll}

Die Daten wurden über aktive (via Snowball-Technik) und passive (Einladungstext mit Link zur Studie, Web-Forum, Offline-Ansprache) Erhebungstechniken online erhoben. Unvollständige Datensätze und solche mit auffälligen Antwortmustern (z.B. alle Werte 0 oder 1) wurden ausgeschlossen, so dass insgesamt $N=1105$ Datensätze in die Analysen eingingen. Die Teilnahme war freiwillig und die TeilnehmerInnen erhielten keine Aufwandsentschädigung. Ihnen wurde 
Tab. 1 Soziodemographische Angaben der Stichprobe $(N=1105)$

\begin{tabular}{|c|c|c|c|}
\hline & & Häufigkeit & Prozent \\
\hline \multirow[t]{2}{*}{ Geschlecht } & Männlich & 151 & 13,7 \\
\hline & Weiblich & 954 & 86,3 \\
\hline \multirow[t]{6}{*}{ Familienstand } & Ledig & 288 & 26,1 \\
\hline & Feste Beziehung & 207 & 18,7 \\
\hline & Verheiratet & 280 & 25,3 \\
\hline & Geschieden & 108 & 9,8 \\
\hline & Verwitwet & 6 & 0,5 \\
\hline & Fehlend & 216 & 19,5 \\
\hline \multirow[t]{5}{*}{ Haushalt } & Alleinlebend & 250 & 22,6 \\
\hline & Mit dem Partner & 300 & 27,1 \\
\hline & Bei den Eltern & 28 & 2,5 \\
\hline & In Wohngemeinschaft & 68 & 6,2 \\
\hline & Fehlend & 459 & 41,5 \\
\hline \multirow{11}{*}{$\begin{array}{l}\text { Höchster } \\
\text { Bildungs- } \\
\text { abschluss }\end{array}$} & Kein Abschluss & 0 & 0 \\
\hline & Hauptschulabschluss & 11 & 1,0 \\
\hline & Realschulabschluss & 53 & 4,8 \\
\hline & Fachhochschulreife & 49 & 4,4 \\
\hline & Abitur & 104 & 9,4 \\
\hline & Berufsschule/Lehre & 102 & 9,2 \\
\hline & Fachhochschulstudium & 115 & 10,4 \\
\hline & Universitätsstudium & 171 & 15,5 \\
\hline & Promotion/Habilitation & 15 & 1,4 \\
\hline & Anderes (z. B. Meisterprüfung) & 26 & 2,4 \\
\hline & Fehlend & 459 & 41,5 \\
\hline \multirow[t]{9}{*}{ Erwerbstätigkeit } & Schüler/in & 12 & 1,1 \\
\hline & Student/in & 88 & 8,0 \\
\hline & Angestellte/r & 278 & 25,2 \\
\hline & Beamtin/er & 39 & 3,5 \\
\hline & Hausfrau/Hausmann & 39 & 3,5 \\
\hline & Selbstständig & 94 & 8,5 \\
\hline & Arbeitssuchend & 58 & 5,2 \\
\hline & Rente/Pension & 38 & 3,4 \\
\hline & Fehlend & 459 & 41,5 \\
\hline
\end{tabular}

jedoch eine Auswertung in Aussicht gestellt. Anonymität wurde zugesichert.

\section{Stichprobe}

Insgesamt haben $N=1105$ Personen an der Studie teilgenommen. Die TeilnehmerInnen waren zwischen 18 und 72 Jahre alt $(M=39,75, S D=11,50) .954(86,3 \%)$ Personen waren weiblich. Für die Prüfung von Unterschiedshypothesen wurde eine nach Alter, Geschlecht, Beruf bzw. nach Bildung gematchte Teilstichprobe verwendet. Hierfür wurden $n=550$ Personen analysiert, 275 je Gruppe. Davon waren $n=482$ (87,6\%) weiblich. Der Altersrange lag in beiden Substichproben zwischen 18 und 70 Jahren $\left(M_{N O N-H S P}=40,91\right.$, $\left.S D_{N O N-H S P}=11,12, M_{H S P}=40,94, S D_{H S P}=11,14\right)$. Detaillierte soziodemographische Angaben können Tab. 1 entnommen werden.

\section{Instrumente}

Persönlichkeits-Stil und Störungsinventar (PSSI; Kuhl und Kazén 2009)

Das PSSI (Kuhl und Kazén 2009) ist ein standardisiertes Selbstbeurteilungsverfahren zur Erfassung der relativen Ausprägung von Persönlichkeitsstilen, welche als nicht-pathologische Entsprechungen der psychiatrischen Persönlichkeitsstörungsdiagnosen des DSM-5 (American Psychiatric Association 2013) und des ICD-10 (Dilling et al. 2000) konzipiert sind. Die Langfassung besteht aus 140 Items, die in 14 Skalen mit jeweils 10 Items unterteilt ist. Jedes Item wird auf einer vierstufigen Likert-Skala (von „stark nicht einverstanden“ bis „stark zustimmend“) angegeben, was zu 0-12 Punkten pro Skala führt, die in T-Werte umgewandelt werden können. Kuhl und Kazén (2009) weisen darauf hin, dass T-Werte oberhalb oder unterhalb einer Standardabweichung des mittleren T-Wertes von 50 (d.h. außerhalb des Bereichs von 40-60) auf individueller Ebene auf das Vorliegen einer Persönlichkeitsstörung hindeuten können, was aber nicht bedeutet, dass diese vorliegt. Dafür wäre eine weiterführende Diagnostik notwendig. Der PSSI verfügt über meist gute Reliabilitäten. Diese variieren zwischen Cronbachs $\alpha=0,73$ bis 0,85 . Die Validität des PSSI wurde in verschiedenen Studien festgestellt und eine gute Konstruktvalidität belegt. In der vorliegenden Studie soll nur die Skala Borderline (BL, spontanborderline) betrachtet werden. Ein Beispielitem für diese Skala wäre: „Meine Gefühle wechseln oft abrupt und impulsiv.“

High Sensitive Person Scale (HSP-Scale; Aron und Aron 1997), German Translation (HSPS-G; Konrad und Herzberg 2017).

Die HSPS-G (Konrad und Herzberg 2017) ist eine übersetzte und modifizierte Skala der HSP-Scale nach Aron und Aron (1997) und beinhaltet 26 Items zur Erfassung von SPS auf einer 5-stufigen Ratingskala von 0 to 4 („0“ „trifft gar nicht zu“; „4“ „trifft völlig zu“). Der Faktor EOE misst die emotionale Reaktivität auf physiologische Stimuli. Während der Faktor AES Reflektion und Bewusstheit erfasst, beschreibt LST unangenehme sensorische Erregung bei äußeren Stimuli. Die Faktoren können aufgrund ihrer hohen Interkorrelation nicht unabhängig voneinander betrachtet werden und beschreiben somit ein übergeordnetes Merkmal, welches auf SPS zurückgeführt werden kann. Alle Skalen können als intern konsistent angesehen werden und gute Retest-Reliabilitäten wurden berichtet (Konrad und Herzberg 2017). Die in der vorliegenden Untersuchung gezeigten Reliabilitäten (Cronbachs alpha) können Tab. 2 entnommen werden. Sie lagen zwischen 0,65 für AES und 0,90 für den HSPS-GS. Die Interkorrelationen zwischen den drei Subskalen liegen zwischen 0,34 für EOE/AES und 0,91 für LST/HSPS-GS und sind in Tab. 2 detailliert dargestellt. 
Tab. 2 Korrelationen zwischen HSPS-G und PSSI-BL und Reliabilitäten der HSPS-G und PSSI-BL

\begin{tabular}{|c|c|c|c|c|c|c|}
\hline & PSSI-BL & PSSI-BL-T & EOE & AES & LST & HSPS_GS \\
\hline \multirow[t]{2}{*}{ EOE } & $0,452^{* * *}$ & $0,464^{\star \star * *}$ & - & - & - & - \\
\hline & $0,379^{\star * *}$ & $0,389^{* * *}$ & - & - & - & - \\
\hline \multirow[t]{2}{*}{ AES } & $0,116^{\star * *}$ & $0,120^{\star \star * *}$ & $0,338^{* \star *}$ & - & - & - \\
\hline & $-0,044$ & $-0,044$ & - & - & - & - \\
\hline \multirow[t]{2}{*}{ LST } & $0,270^{* * *}$ & $0,277^{\star \star \star *}$ & $0,610^{* * *}$ & $0,453^{* * *}$ & - & - \\
\hline & 0,007 & 0,007 & - & - & - & - \\
\hline HSPS-GS & $0,371^{\star \star *}$ & $0,381^{* * *}$ & $0,845^{* * *}$ & $0,602^{* \star *}$ & $0,914^{* \star *}$ & - \\
\hline Reliabilität & 0,85 & - & 0,82 & 0,65 & 0,87 & 0,90 \\
\hline
\end{tabular}

Tab. 3 Ergebnisse des Welch-Test zwischen den Gruppen NON-HSP und HSP

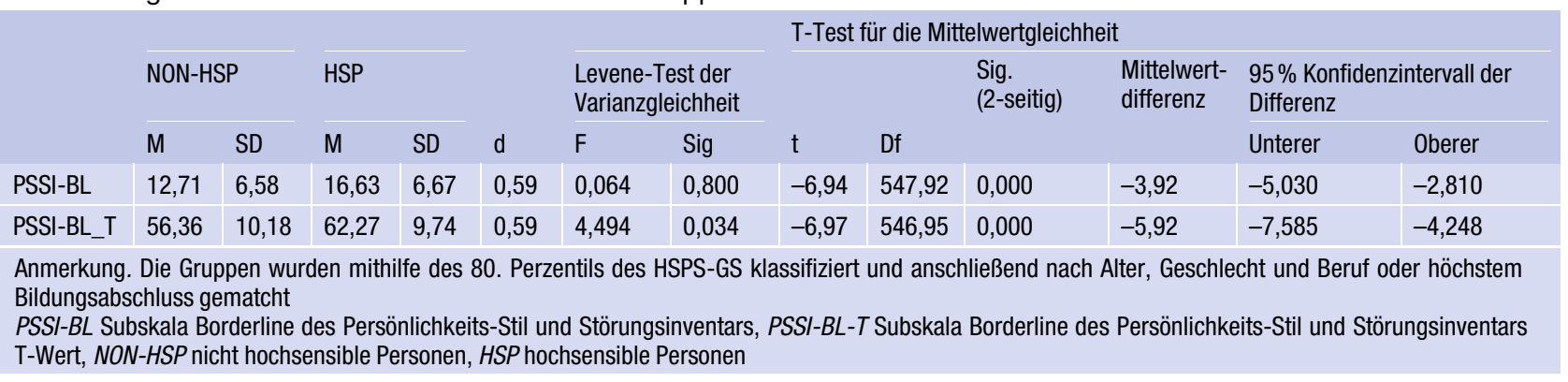

\section{Statistische Analyse}

Um Unterschiede in der BPS-Symptomatik zwischen hochsensiblen und nicht-hochsensiblen Personen zu untersuchen, wurde eine Gruppenbildung vorgenommen. Dabei wurden Männer und Frauen zunächst getrennt betrachtet, da sie sich hinsichtlich ihrer Mittelwerte in SPS unterscheiden. Nach Kagan et al. (1994) lassen sich ca. $20 \%$ der Gesamtbevölkerung als hochsensibel beschreiben. Daher wurde das 80. Perzentil des HSPS-Gesamtwertes (HSPS-GS) verwendet, um die Gruppe der Hochsensiblen zu bestimmen. Damit ergab sich für die Gruppe der hochsensiblen Männer ein Cut-off von $\geq 81$ im HSPS-GS und für die Gruppe der hochsensiblen Frauen ein cut-off von $\geq 88$ ebenfalls im HSPS-GS. Den so ausgewählten Probanden für die Gruppe der Hochsensiblen wurde nun per Zufall eine nicht-hochsensible Person gegenübergestellt, gematcht nach Alter, Geschlecht und Beruf bzw. höchster Bildungsabschluss. Damit ergaben sich für die weiteren Analysen mittels Welch-Test zwei sehr homogene Gruppen. Zur Bestimmung von Prädiktoren für den PSSI-BL-Wert wurde zunächst eine einfache Regression mit dem HSP-GS als Prädiktorvariable gerechnet. Im zweiten Schritt wurde geprüft, welcher der drei Subfaktoren (EOE, AES, LST) von SPS den Score auf der PSSI-BL-Skala am besten vorhersagt. Dafür wurde die schrittweise Regression gewählt. Bei diesem Verfahren wurde systematisch die signifikanteste Variable automatisch hinzugefügt und die am wenigsten signifikante Variable entfernt. In einem weiteren Schritt wurden die Variablen Alter und Geschlecht noch hinzugefügt, um den Einfluss zu prüfen. Dieses Verfahren wurde gewählt, weil noch keine empirischen Belege zur Verfügung standen, auf die man sich bei der Analyse hätte stützen können.

\section{Ergebnisse}

\section{Korrelationsanalyse}

Die Ergebnisse der Korrelationsanalyse können Tab. 2 entnommen werden. Der Faktor EOE der HSPS-G zeigt eine mittlere Korrelation mit der PSSI-BL Subskala. Die HSPS-G Subskalen EOE und LST sind sehr schwach bis schwach mit der BL-Subskala des PSSI korreliert. Ebenso zeigt sich eine mittlere Korrelation mit dem HSPS-GS und der BL-Subskala. Nach Auspartialisierung der jeweils anderen beiden Faktoren korreliert der Faktor EOE immer noch mittel mit der BL-Subskala. Der Faktoren AES und LST korrelieren nicht mehr mit der BL-Subskala.

\section{Gruppenvergleich}

Die Ergebnisse der Mittelwertvergleiche werden in Tab. 3 berichtet. Wie dort zu entnehmen, berichten hochsensible Personen auf der BL-Subskala des PSSI 
Tab. 4 Regressionsanalyse

\begin{tabular}{|c|c|c|c|c|c|c|c|c|}
\hline AV & & $\mathrm{F}$ & Df & $p$ & Adj. $R^{2}$ & Sig. Veränderung in $\mathrm{F}(p)$ & $\mathrm{t}$ & Standardisiertes $\beta$ \\
\hline \multirow[t]{2}{*}{ PSSI-BL-T } & Modell 1 & & & & & & & \\
\hline & HSPS-GS & 187,09 & 1 & 0,000 & 0,144 & 0,000 & 13,68 & $0,38^{\star \star \star}$ \\
\hline \multirow[t]{2}{*}{ PSSI-BL-T } & Modell 1 & & & & & & & \\
\hline & EOE & 302,51 & 1 & 0,000 & 0,214 & 0,000 & 17,39 & $0,46^{\star \star \star}$ \\
\hline \multirow[t]{5}{*}{ PSSI-BL-T } & Modell 1 & & & & & & & \\
\hline & EOE & 303,32 & 1 & 0,000 & 0,214 & 0,000 & 17,42 & $0,46^{\star \star \star}$ \\
\hline & Modell 2 & & & & & & & \\
\hline & EOE & 220,11 & 2 & 0,000 & 0,283 & 0,000 & 18,81 & $0,48^{\star \star \star}$ \\
\hline & Alter & - & - & - & - & - & $-10,38$ & $-0,26^{\star \star \star}$ \\
\hline
\end{tabular}

AV Abhängige Variable, PSSI-BL-T Subskala Borderline des Persönlichkeits-Stil und Störungsinventars T-Wert, EOE Leichte Erregbarkeit, HSPS-GS HSPGesamtwert

${ }^{\star \star \star} p \leq 0,001,{ }^{* \star} p \leq 0,01,{ }^{*} p \leq 0,05$

höhere Werte im Vergleich zu den Nicht-Hochsensiblen Personen. Der Effekt ist mittelgroß ausgeprägt.

\section{Regressionsanalyse}

Aus Tab. 4 können die Ergebnisse der Regressionsanalyse entnommen werden. Da bislang noch keine Vergleichsdaten vorliegen, wurden zuerst eine einfache Regression und dann eine schrittweise Regressionsanalyse gerechnet. Die Ergebnisse zeigen, dass der HSPS-GS 14,4\% der Varianz des PSSI-BL-T erklärt. Die schrittweise Regressionsanalyse ergab, dass der Subskala EOE die größte Bedeutung zukommt. Sie klärt mit $21,4 \%$ den größten Anteil der Varianz am PSSIBL-T auf. Die Subfaktoren AES und LST wurden aus dem Modell entfernt, weil sie keinen Beitrag zur Vorhersage von PSSI-BL-T leisten. Die Prüfung der Kontrollvariablen Alter und Geschlecht zeigt ferner einen Einfluss des Alters. Diese Variable erklärt weitere 6,9\% der Varianz.

Zusammengefasst zeigen die Ergebnisse, dass hauptsächlich die Subskala EOE eine BL-Symptomatik vorhersagt, der Effekt aber auch nicht ganz unabhängig vom Alter ist und gerade auch bei jüngeren Personen eine Rolle spielt.

\section{Diskussion}

Ziel der vorliegenden Studie war es, den Zusammenhang zwischen SPS und einer BL-Symptomatik zu untersuchen und Gemeinsamkeiten und Unterschiede zu eruieren.

Die Korrelationsanalyse zeigt einen mittleren $\mathrm{Zu}$ sammenhang zwischen der SPS-Subskala EOE und der PSSI-Subskala BL, der auch nach Auspartialisierung der beiden SPS-Subskalen AES und LST aufgrund ihrer hohen Interkorrelationen, erhalten bleibt. Die Subskala EOE bildet die Reaktivität auf physische Reize ab, also genau den Aspekt, der beide Konstrukte verbindet und somit auf phänotypischer Ebene zu Verwechslungen führen könnte. Die SPS-Subskalen AES und LST zeigen keine Zusammenhänge zur PSSISubskala BL. Damit grenzen diese sich wiederum von einer BL-Symptomatik ab und sprechen für eine diskriminante Validität. HSPS-GS und PSSI-BL weisen ebenfalls eine mittlere Korrelation auf. Die Ergebnisse decken sich zum Teil mit der Studie von Meyer et al. (2005), in der eine ähnlich hohe Korrelation $(r=0,43)$ zwischen HSP-Scale und der Borderline Skala des SCID-II berichtet wird. Allerdings wurde hier nur der HSP-Gesamtscore berücksichtigt, so dass zu den Subskalen der HSP-Scale keine differenzierteren Angaben gemacht werden konnten. Die Ergebnisse der HSPS-Subskalen decken sich aber mit Ergebnissen aus anderen Studien zur psychischen Symptombelastung. So waren beispielsweise Autismus, Angst und Depression mit EOE assoziiert (z. B. Liss et al. 2008; Konrad und Herzberg 2017). Ebenso wird EOE mit dem 5-HTTLPR-Polymorphismus (5-HTT gene linked polymorphic region) in Verbindung gebracht (Licht et al. 2011). Dieses Serotonin-Transporter-Gen weist wiederum Zusammenhänge zu höheren Werten in Neurotizismus auf, ein Persönlichkeitstrait, der sowohl bei SPS (z. B. Ahadi und Basharpoor 2010; Aron und Aron 1997; Smolewska et al. 2006) als auch bei BPS (z. B. Morey und Zanarini 2000) eine Rolle spielt.

Der Vergleich der Mittelwertunterschiede in den Gruppen HSP und NON-HSP hat gezeigt, dass Personen der Gruppe HSP auf der PSSI-BL-Skala höhere Werte berichteten im Vergleich zur NON-HSPGruppe. Die Effekte sind mittelgroß ausgeprägt. Auch diese Ergebnisse sind mit Studien zur psychischen Symptombelastung konform. Auch bei Konrad und Herzberg (2017) zeigte die HSP-Gruppe höhere Werte in psychischer Symptombelastung, gemessen mit der Brief Symptom Checklist (BSCL; Franke 2016) im Vergleich zur Gruppe der NON-HSP.

Die Ergebnisse der Regressionsanalyse zeigen, dass lediglich die hohe emotionale Ansprechbarkeit (EOE) Varianz an der PSSI-BL Skala erklärt. Die Varianzaufklärung lag bei $21,4 \%$. Durch Berücksichtigung des Alters konnten weitere 6,9\% Varianz aufgeklärt werden. Das negative Betagewicht spricht dafür, dass die hohe emotionale Reagibilität besonders in jungen Jahren einen Borderline-Persönlichkeitsstil vorhersagt, was damit konform ist, dass die Erkrankung 
mit zunehmendem Alter seltener auftritt (Distel et al. 2008).

\section{Limitationen und Implikationen}

Da es sich um eine Online-Studie handelte, konnte die Zusammensetzung der Stichprobe nicht kontrolliert werden und ist nicht repräsentativ. Aber eine echte zufallsgesteuerte Stichprobenauswahl ist schwer bzw. unmöglich. Deshalb wurde versucht über aktive (Snowball-Technique) und passive (Einladungstext mit Link auf Website oder Webforum, Offline-Ansprache) Auswahl von Probanden die Untersuchung breit zu streuen und eine große Stichprobe zu erheben, um Stichprobeneffekte zu reduzieren. Auch hat sich gezeigt, dass online erhobene Stichproben eine deutlich größere Diversität, als offline erhobene Stichproben aufweisen (Gosling et al. 2004).

Ferner ist nicht auszuschließen, dass der Zusammenhang zwischen beiden Phänomenen durch Antwortstile oder übergreifende Merkmale zustande gekommen ist. Auch der Anteil der Frauen war in der vorliegenden Untersuchung sehr hoch. Ferner sind aber weitere Studien notwendig, die insbesondere klinische BPS-Gruppen, hochsensible Personen und Kontrollgruppen (mit niedrigen Ausprägungen in BPS, SPS und psychischer Symptombelastung) untersuchen, um Gemeinsamkeiten und Unterschiede zwischen den Gruppen herauszuarbeiten. Künftig sind auch Längsschnittuntersuchungen notwendig, um Kausalitäten zu prüfen. Als Implikation für die Praxis sollte bei einer BPS geprüft werden, ob eventuell auch eine Hochsensibilität mit vorliegen kann, da beide Phänomene Überschneidungspunkte aufweisen können. Dies sollte durch den Therapeuten gegenüber dem Patienten auch entsprechend kommuniziert werden, weil das Wissen über eine eventuell vorliegende Hochsensibilität (wahrscheinlich auch bei BPS-PatientInnen) bereits Erleichterung verschafft. Betroffene fühlen sich meist ihr Leben lang unverstanden und haben ein tiefgreifendes Gefühl „anders“ zu sein, was im Grunde auf beide Phänomene zutrifft. Mit diesem Wissen erhalten Betroffene beider Gruppen eine neue Identität bzw. einen neuen Bezugsrahmen, der sie nicht mehr zwingt, eine Rolle spielen zu müssen, welche nicht für sie zugeschnitten ist. Um $\mathrm{zu}$ verstehen, wo die eigenen Grenzen sind, ist es wichtig den eigenen Körper und seine Signale $\mathrm{zu}$ kennen und sich selbst einzugestehen, dass die Betroffenen mehr Zeiten der Ruhe und Entspannung benötigen als der Durchschnitt, und sich diese Ruhezeiten auch zugestehen. Hierfür ist es notwendig nicht über Vergleiche mit anderen zu gehen, sondern seine eigene Balance zwischen Aktivität und Rückzug zu finden. Wichtig ist auch die eigene Vergangenheit vor dem Hintergrund der eigenen $\mathrm{HS}$ zu verstehen und dadurch das eigene Leben aus einer neuen Perspektive betrachten zu können und so zu einer „inneren“ Heilung zu kommen. Es empfehlen sich achtsam- keitsbasierte Interventionen (z.B. Berking und von Känel 2007; Baer 2003). Diese können dazu beitragen, eine Vielzahl von psychischen Gesundheitsproblemen $\mathrm{zu}$ lindern und die psychische Funktionsfähigkeit zu verbessern (Baer 2003). Achtsamkeitsbasierte Interventionen scheinen konzeptionell mit vielen anderen empirisch gestützten Behandlungsansätzen konsistent zu sein und können eine Akzeptanztechnologie bieten, die die Technologie der Veränderung ergänzt, die bei den meisten kognitiv-behavioralen Verfahren zum Einsatz kommt (Linehan 1993).

Funding Open Access funding enabled and organized by Projekt DEAL.

Interessenkonflikt S. Konrad gibt an, dass kein Interessenkonflikt besteht.

Open Access Dieser Artikel wird unter der Creative Commons Namensnennung 4.0 International Lizenz veröffentlicht, welche die Nutzung, Vervielfältigung, Bearbeitung, Verbreitung und Wiedergabe in jeglichem Medium und Format erlaubt, sofern Sie den/die ursprünglichen Autor(en) und die Quelle ordnungsgemäß nennen, einen Link zur Creative Commons Lizenz beifügen und angeben, ob Änderungen vorgenommen wurden.

Die in diesem Artikel enthaltenen Bilder und sonstiges Drittmaterial unterliegen ebenfalls der genannten Creative Commons Lizenz, sofern sich aus der Abbildungslegende nichts anderes ergibt. Sofern das betreffende Material nicht unter der genannten Creative Commons Lizenz steht und die betreffende Handlung nicht nach gesetzlichen Vorschriften erlaubt ist, ist für die oben aufgeführten Weiterverwendungen des Materials die Einwilligung des jeweiligen Rechteinhabers einzuholen.

Weitere Details zur Lizenz entnehmen Sie bitte der Lizenzinformation auf http://creativecommons.org/licenses/by/4. $0 /$ deed.de.

\section{Literatur}

Ahadi, B., \& Basharpoor, S. (2010). Relationship between sensory processing sensitivity, personality dimensions and mentalhealth. Journal of Applied Sciences, 10(7), 570-574.

American, P., \& Association (2013). Diagnostic and statistical manual of mental disorders (5. Aufl.). Washington: American Psychiatric Association.

American, P., \& Association (2015). Diagnostisches und Statistisches Manual Psychischer Störungen DSM-5. Göttingen, Bern, Wien: Hogrefe.

Aron, E. N., \& Aron, A. (1997). Sensory-processing sensitivity and its relation to introversion and emotionality. Journal of Personality and Social Psychology, 73(2), 345-368.

Aron, E. N., Aron, A., \& Davies, K. M. (2005). Adult shyness: the interaction of temperamental sensitivity and an adverse childhood environment. Personality and Social Psychology Bulletin, 31(2), 181-197.

Aron, E. N., Aron, A., \& Jagiellowicz, J. (2012). Sensory processing sensitivity: a review in the light of the evolution of biological responsivity. Personality and Social Psychology Review, 16(3), 262-282.

Assary, E., Zavos, H.M., Krapohl, E., Keers, R., \& Pluess, M. (2020). Genetic architecture of Environmental Sensitivity reflects multiple heritable components: a twin study with adolescents. Molecular Psychiatry, 1-9. 
Baer, R.A. (2003). Mindfulness training as a clinical intervention: a conceptual and empirical review. Clinical psychology: Science and practice, 10(2), 125-143.

Belsky, J. (1997). Variation in susceptibility to environmental influence: an evolutionary argument. Psychological inquiry, 8(3), 182-186.

Belsky, J., \&Pluess, M. (2009). Beyond diathesis stress: differential susceptibility to environmental influences. Psychological Bulletin, 135(6), 885-908.

Berking, M., \& von Känel, M. (2007). Achtsamkeitstraining als psychotherapeutische Interventionsmethode. $P P m P$ Psychotherapie. Psychosomatik. Medizinische Psychologie, 57(03/04), 170-177.

Dilling, H., Mombour, W., Schmidt, M., \& Schulte-Markwort, E. (2000). Weltgesundheitsorganisation: Internationale Klassifikation psychischer Störungen: ICD-10. klinisch-diagnostische Leitlinien (4).Göttingen, Toronto, Seattle: Huber

Distel, M., Trull, T., Derom, C., Thiery, E., Grimmer, M., Martin, N., et al. (2008). Heritability of borderline personality disorder features is similar across three countries. Psychological medicine, 38(9), 1219-1229.

Eaton, N.R., Krueger, R.F., Keyes, K.M., Skodol, A.E., Markon, K.E., Grant, B. F, et al. (2011). Borderline personality disorder co-morbidity: relationship to the internalizing-externalizing structure of common mental disorders. Psychological medicine, 41(5), 1041-1050.

Ellis, B.J., Boyce, W.T., Belsky, J., Bakermans-Kranenburg, M.J., \& Van IJzendoorn, M.H. (2011). Differential susceptibility to the environment: An evolutionary-neurodevelopmental theory. Development and psychopathology, 23(1), 7-28.

Evers, A., Rasche, J., \& Schabracq, M. J. (2008). High sensoryprocessing sensitivity at work. International Journal of Stress Management, 15(2), 189-198.

Franke, G. H. (2016). BSCL. Brief Symptom Checklist. Manual. Göttingen: Hogrefe, in Vorbereitung.

Gosling, S.D., Vazire, S., Srivastava, S., \& John, O. P. (2004). Should we trust web-based studies? A comparative analysis of six preconceptions about internet questionnaires. American Psychologist, 59, 93-104. https://doi.org/ 10.1037/0003-066X.59.2.93.

Greven, C. U., Lionetti, F., Booth, C., Aron, E. N., Fox, E., Schendan, H. E., Homberg, J. et al. (2019). Sensory Processing Sensitivity in the context of Environmental Sensitivity: A critical review and development of research agenda. Neuroscience \& Biobehavioral Reviews, 98, 287-305.

Hinterberger, T., Galuska, D., \& Galuska, J. (2019). Der SV12: Entwicklung eines klinischen Inventars zur Erfassung von Sensibilität und deren Verarbeitungsproblematiken. Complementary medicine research, 26(4), 240-249.

Kagan, J., Snidman, N., Arcus, D., \& Reznick, J.S. (1994). Galen's prophecy: Temperament in human nature. New York: Basic Books.

Khayal, S., Kohl, S., \&Niehaus, M. (2016). BerufsbezogeneKompetenzen von Personen mit einer Borderline Persönlich- keitsstörung (BPS): Anregungen für die Berufsbildung. In A. Zoyke \& K. Vollmer (Hrsg.), Inklusion in der Berufsbildung: Befunde - Konzepte - Diskussionen (S. 163-177). Bonn: Bundesinstitut für Berufsbildung.

Konrad, S., \& Herzberg, P.Y. (2017). Psychometric properties and validation of a German High Sensitive Person Scale (HSPS-G). European Journal of Psychological Assessment. https://doi.org/10.1027/1015-5759/a000411.

Kuhl, J., \& Kazén, M. (2009). Persönlichkeits-Stil-und StörungsInventar: PSSI; Manual. Göttingen: Hogrefe.

Lammers, C.-H., Röpke, S., \& Dulz, B. (2007). Selbstwert und Borderline-Persönlichkeitsstörung. PTT-Persönlichkeitsstörungen: Theorie und Therapie, 11(1), 31-40.

Licht, C. L., Mortensen, E. L., \& Knudsen, G. M. (2011). Association between sensory processing sensitivity and the serotonin transporter polymorphism 5-HTTLPR short/short genotype. Paper presented at the Biological Psychiatry.

Linehan, M. M. (1993). Cognitive-behavioral treatment of borderline personality disorder. NewYork: Guilford.

Lionetti, F., Aron, A., Aron, E. N., Burns, G.L., Jagiellowicz, J., \& Pluess, M. (2018). Dandelions, tulips and orchids: evidence for the existence of low-sensitive, mediumsensitive and high-sensitive individuals. Translational psychiatry, 8(1), 24.

Liss, M., Mailloux, J., \& Erchull, M. J. (2008). The relationships between sensory processing sensitivity, alexithymia, autism, depression, and anxiety. Personality and Individual Differences, 45(3), 255-259.

Meyer, B., Ajchenbrenner, M., \& Bowles, D. P. (2005). Sensory sensitivity, attachment experiences, and rejection responses among adults with borderline and avoidant features. Journal of Personality Disorders, 19(6), 641-658.

Morey, L.C., \& Zanarini, M.C. (2000). Borderline personality: traits and disorder. Journal of Abnormal Psychology, 109(4), 733.

Russell, J. J., Moskowitz, D., Zuroff, D. C., Sookman, D., \& Paris, J. (2007). Stability and variability of affective experience and interpersonal behavior in borderline personality disorder. Journal of Abnormal Psychology, 116(3), 578.

Smolewska, K. A., McCabe, S. B., \& Woody, E.Z. (2006). A psychometric evaluation of the highly sensitive person scale: the components of sensory-processing sensitivity and their relation to the BIS/BAS and "big five". Personality and Individual Differences, 40(6), 1269-1279.

Stepp, S.D., Pilkonis, P.A., Yaggi, K.E., Morse, J.Q., \& Feske, U. (2009). Interpersonal and emotional experiences of social interactions in borderline personality disorder. The Journal of nervous and mental disease, 197(7), 484.

Torgersen, S., Lygren, S., Øien, P. A., Skre, I., Onstad, S., Edvardsen, J., et al. (2000). A twin study of personality disorders. Comprehensive psychiatry, 41(6), 416-425.

Hinweis des Verlags Der Verlag bleibt in Hinblick auf geografische Zuordnungen und Gebietsbezeichnungen in veröffentlichten Karten und Institutsadressen neutral. 